\title{
Conditions de formation d'eaux colorées toxiques sur le plateau continental Manche - Atlantique; Cas de Gyrodinium cf. aureolum
}

P. Le Corre(1), S. L'Helguen ${ }^{(1)}$, P. Morin ${ }^{(2)}$ et J.L. Birrien ${ }^{(2)}$

(1) Laboratoire d'Océanographie Chimique, Université de Bretagne Occidentale, 6, Avenue V. Le Gorgeu, 29287 Brest Cedex, France.

(2) Observatoire Océanologique de Roscoff, CNRS \& Université P. et M. Curie, B.P. 74, 29682 Roscoff Cedex, France.

Résumé. - Gyrodinium cf. aureolum a donné lieu ces dernières années à de nombreux phénomènes d'eaux colorées dans les eaux côtières de l'Europe du Nord-Ouest avec parfois des incidences graves sur le fonctionnement des systèmes. Deux séries d'observations menées en 1983 et 1986 sur deux systèmes différents du plateau continental Manche - Atlantique ont permis de préciser les conditions de prolifération de l'espèce. Nos mesures des flux d'absorption (technique ${ }^{15} \mathrm{~N}$ ) montrent que l'azote nécessaire au développement massif de Gyrodinium se trouve essentiellement sous forme d'ammonium. Ce composé est libéré par des processus intenses de régénération qui correspondent à la présence dans le système de fortes teneurs en matière organique détritique. Ce matériel détritique a pu être formé sur place (c'est le cas du front d'Ouessant); il peut aussi avoir été apporté de l'extérieur et résulter d'une perturbation du système (cas de la Baie de Douarnenez). II est alors clair que Gyrodinium cf. aureolum peut se développer dans des systèmes où le stock apparent d'éléments nutritifs est réduit.

Abstract. - Gyrodinium cf. aureolum was responsible for the numerous instances of red tide recorded in the coastal waters of the North-Western Europe, often accompanied by serious disturbances in the functioning of the ecosystems. Independent measurements of nitrogen fluxes measured in two different ecosystems on the Western English Channel continental shelf showed that massive blooms of Gyrodinium cf. aureolum are sustained essentially by ammonium. High concentrations of detrital organic matter coupled with intense in situ regeneration is responsible for a continuous supply of ammonium necessary for the bloom. The detrital substrate can be of either in situ origin, as in the case of the Ushant front, or of an origin extraneous to the system, as in the Douarnenez Bay. Thus blooms of Gyrodinium ct. aureolum can occur even when apparent ambient nutrient levels are very low. 


\section{INTRODUCTION}

Le dinoflagellé Gyrodinium cf. aureolum, observé il y a quelques trente ans sur la côte Est des États-Unis (Hulburt, 1957), a été identifié pour la première fois en 1966 en Europe du Nord, alors qu'il formait un phénomène intense d'eau colorée au sein des eaux côtières norvégiennes (Braarud et Heimdal, 1970). Depuis cette époque, de nombreuses poussées de Gyrodinium cf. aureolum ont été détectées dans les eaux européennes que ce soit en Norvège (Tangen, 1977; Dahl et al., 1982), en Suède (Lindhal, 1983), autour des lles Britanniques (Ballantine et Smith, 1973; Helm et al., 1974; Ottway et al., 1979; Boalch, 1979; Pybus, 1980 entre autres) ou encore sur le littoral français (Grall, 1977; Lassus, 1984; Morin et al., 1989). Elles ont souvent été accompagnées de mortalité d'organismes pélagiques et benthiques (Tangen, 1977; Foster, 1979; Griffiths et al., 1979; Dahl et al., 1982) en raison de la toxicité de l'espèce ou comme le résultat d'une déficience en oxygène des masses d'eau (Tangen, 1977). Gyrodinium cf. aureolum s'est aussi avéré très abondant à différentes occasions, plus au large, au voisinage des fronts thermiques qui s'établissent à la fin du printemps en Mer Celtique et en Manche Occidentale (Pingree et al. 1975; Pingree et al. 1977; Holligan, 1979). Selon Parker et al. (1982), Gyrodinium cf. aureolum se développerait d'abord dans les eaux du large et les structures frontales pourraient alors jouer un rôle important dans la dissémination de l'espèce dans les systèmes les plus côtiers (Ayres et al., 1982). En dépit d'un grand nombre d'observations (pour une revue détaillée, on se reportera à la synthèse bibliographique de Partensky et Sournia, 1986), les conditions de développement de Gyrodinium cf. aureolum jusqu'au stade d'eaux colorées demeurent mal connues. II se pose en particulier le problème d'une source suffisante d'azote pour expliquer un tel développement phytoplanctonique. Les teneurs en azote mesurées sur place ne peuvent en effet rendre compte des concentrations extrêmes en phytoplancton qui ont été souvent observées. Le développement de Gy. rodinium cf. aureolum peut résulter du transfert des nitrates de la couche de fond souvent riche en éléments nutritifs (Pingree et al., 1977; Holligan et al., 1983); ces apports sont néanmoins limités par la thermocline et semblent insuffisants pour expliquer les fortes biomasses observées.

La Baie de Douarnenez (fig. 1) a donné lieu lors de l'été 1983 à une poussée intensive de Gyrodinium. Une série de douze campagnes réalisées de mars 1983 à février 1984 et dont l'objectif initial était d'étudier l'incidence du front interne de l'Iroise sur la production phytoplanctonique de ce secteur côtier ont permis d'examiner les différentes séquences du phénomène (Birrien et al., 1991). Les résultats mettent en avant de manière indirecte (voir ci-après) l'existence de flux d'azote régénéré qui semblent suffisamment importants pour expli- 


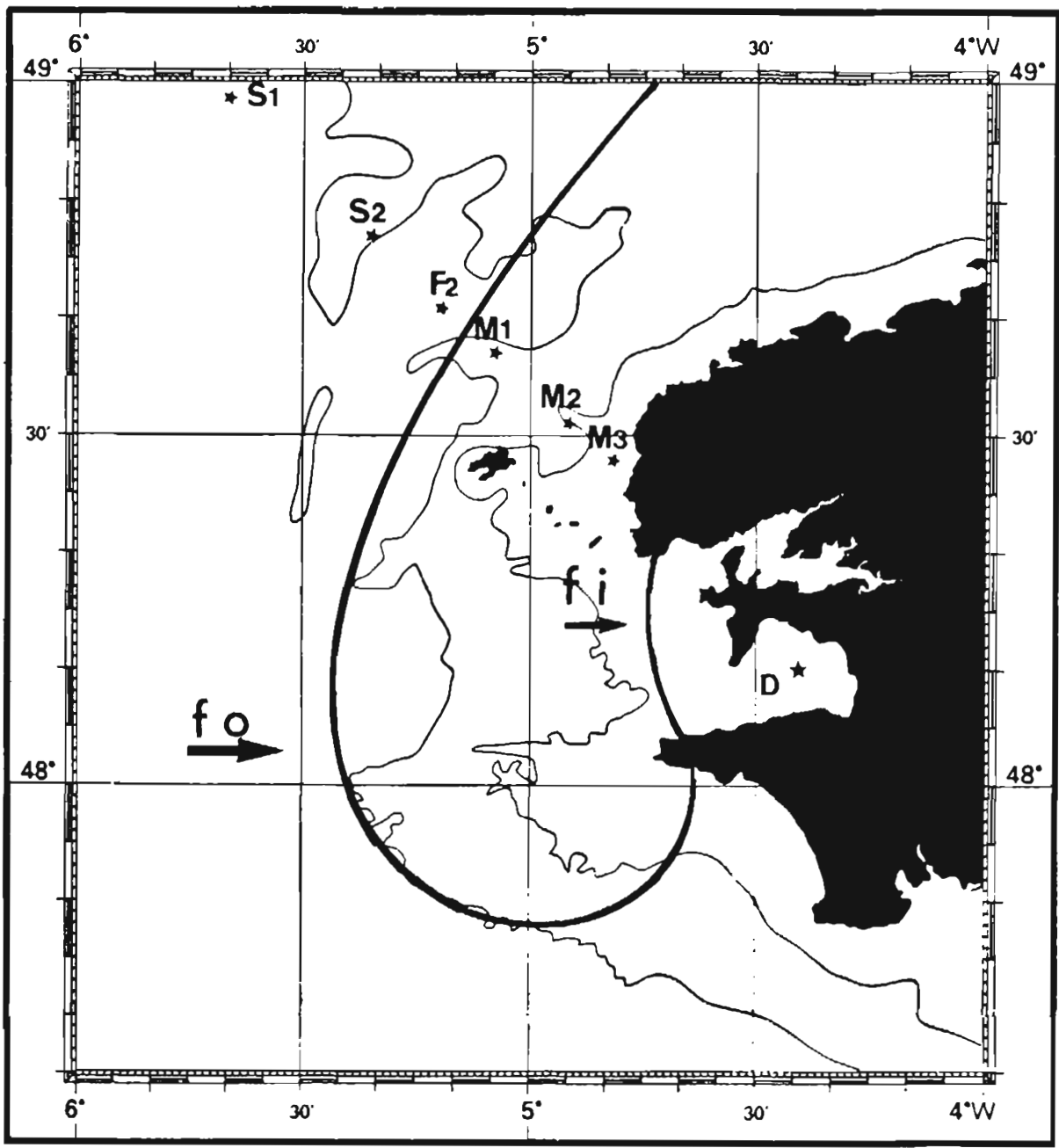

Fig. 1. - Zone d'étude et emplacement des stations lors des campagnes à la mer: F.O. Front thermique d'Ouessant, F.I. Front Interne de l'Iroise. Baie de Douarnenez (1983): station D. Campagne Iroise (1986): stations $S_{1}, S_{2}, F_{2}, M_{1}, M_{2}, M_{3}$.

Fig. 1. - Study area and location of the stations with: F.O. Ushant thermal front, F.I. Iroise Inner Front. Bay of Douarnenez (1983): point $D$. Iroise cruise (1986): stations $S_{1}, S_{2}, F_{2}, M_{1}, M_{2}, M_{3}$.

quer la prolifération sur place de Gyrodinium $c f$. aureolum. L'idée de l'existence d'une source régénérée abondante en azote qui assurerait le développement de Gyrodinium a été examinée en juillet 1986 au cours de 
la campagne lroise dans le secteur du front thermique d'Ouessant (pour l'emplacement des stations, voir fig. 1). Les flux d'absorption et de régénération de l'azote ont été mesurés par la technique ${ }^{15} \mathrm{~N}$ lors d'un développement intense de Gyrodinium cf. aureolum au voisinage du front (L'Helguen, 1991). Notre objectif dans ce texte est, en prenant compte ces deux séries d'observations, d'analyser les conditions de prolifération de Gyrodinium ct. aureolum sur le plateau continental Manche - Atlantique. L'accent est mis sur l'origine et la nature de l'azote utilisé lors de la formation des eaux colorées.

\section{MATÉRIEL ET MÉTHODES}

A la station D en Baie de Douarnenez, les prélèvements ont été réalisés à l'aide de bouteilles de type Niskin (de 5 I de contenance). Les températures ont été déterminées par des thermomètres à renversement de type Richter et Wiese (précision de lecture $\pm 0.01^{\circ} \mathrm{C}$ ). Les échantillons de salinité ont été analysés au laboratoire sur un salinomètre Guildine Autosal modèle 8400 avec une précision de \pm 0.005 P.S.U. Les échantillons de sels nutritifs conservés congelés (à $-25^{\circ} \mathrm{C}$ ) dans des flacons en polyéthylène ont été analysés au laboratoire en analyse automatique selon les procédures décrites par Tréguer et Le Corre (1975). La chlorophylle a a été déterminée selon la méthode fluorimétrique de Yentsch et Menzel (1963). Dans le cas du front d'Ouessant, les prélèvements ont été réali- sés par un système bathysonde et l'ensemble des mesures effectuées à bord selon les techniques citées précédemment. Les flux d'absorption des composés azotés $\left(\mathrm{NO}_{3}^{-}, \mathrm{NH}_{4}^{+}\right)$et de régénération $\left(\mathrm{NH}_{4}^{+}\right)$ont été déterminés en appliquant la méthode du traceur ${ }^{15} \mathrm{~N}$ selon les protocoles décrits par L'Helguen (1991).

\section{RÉSULTATS ET DISCUSSION}

\section{Conditions de développement de Gyrodinium cf. aureolum (1983) \\ au voisinage du front interne de I'Iroise (Station $D$ en Baie de Douarnenez)}

\section{Variations de la structure hydrologique}

Homogènes en hiver et au début du printemps (fig. 2a et b), les masses d'eau de la Baie de Douarnenez montrent dès le mois de mai une forte stratification qui se maintient par la suite durant tout l'été (fig. 2c). Elle présente dans le temps deux origines différentes; au printemps, elle est de nature haline: elle est alors engendrée par les fortes dessalures qui apparaissent à cette époque dans la baie (fig. 2b). Ces dessalures correspondent à une pénétration d'eaux dessalées formées à l'extérieur sur le plateau continental. Ces eaux trouvent leur origine dans le Sud Bretagne sous l'influence prépondérante des eaux de la Loire alors que le printemps a été particulièrement pluvieux (Birrien, 1987). Elles sont épuisées en 

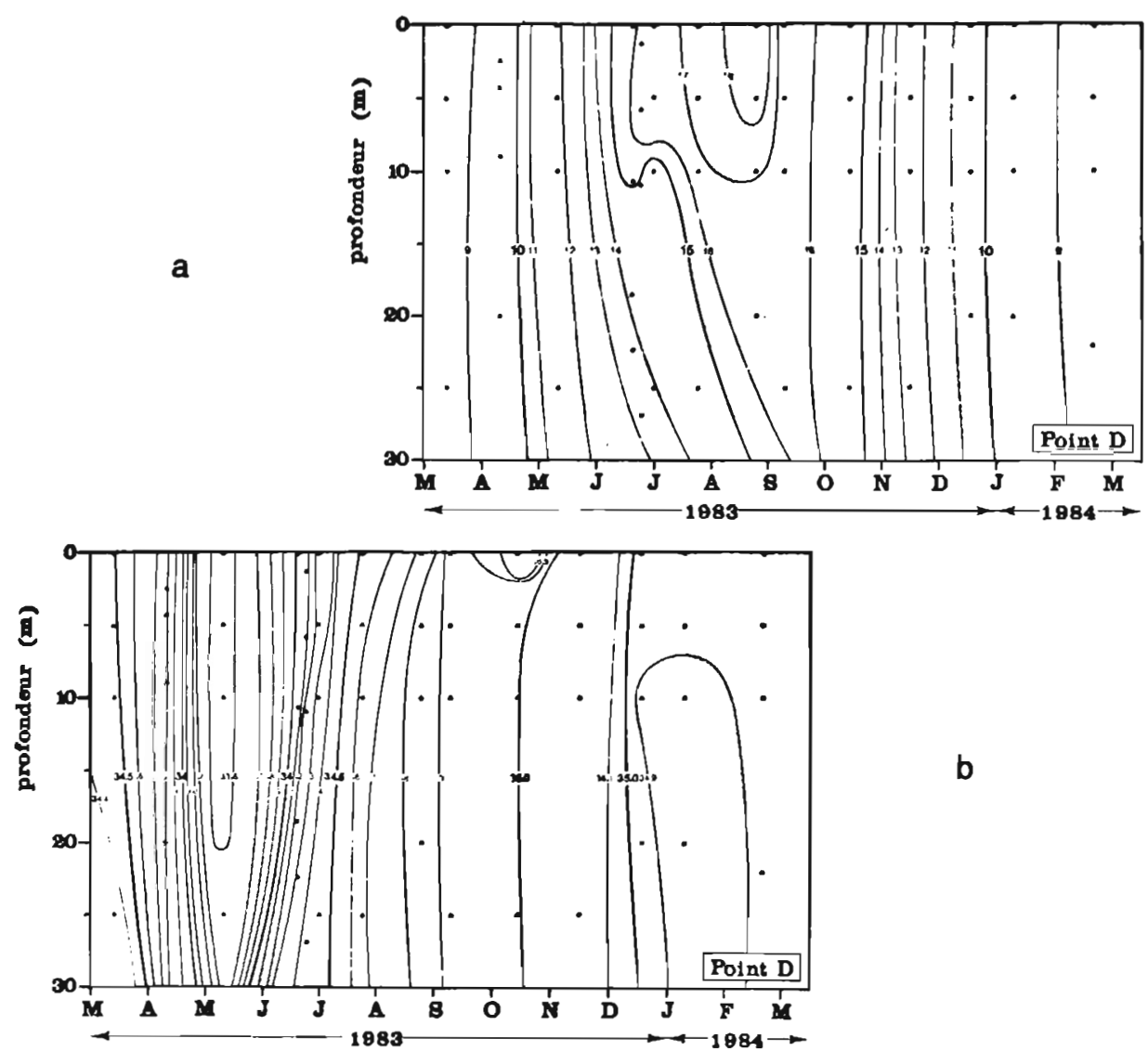

b

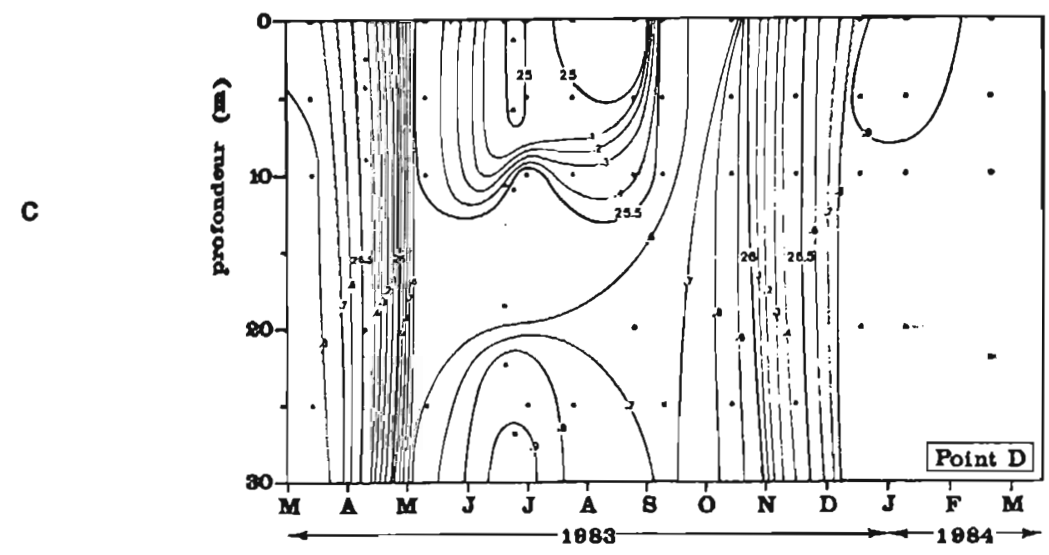

Fig. 2. - Distribution verticale en 1983 à la station $D$. a. température $\left({ }^{\circ} \mathrm{C}\right)$. La colonne d'eau est bien stratifiée de juin à septembre. b. salinité (en P.S.U.). Des faibles salinités sont mises en évidence en surface en mai. c. densité (sigma T).

Fig. 2. - Vertical distribution in 1983 at station D. a. temperature $\left({ }^{\circ} \mathrm{C}\right)$. The water column is well statified from june to september. b. salinity (P.S.U.). Low-salinity waters are detected in surface in may. c. density (sigma $\mathrm{T}$ ). 
éléments nutritifs (fig. 3a et b); leur transit sur le plateau continental a été assez long pour permettre une assimilation totale des nutriments. II est à remarquer qu'elles introduisent dans la baie des quantités importantes d'azote et de carbone sous forme organique (fig. 4). En juin, la stratification thermique prend le relais de la stratification haline. La thermocline est peu profonde (fig. 2a) et la pycnocline (fig. 2c) se maintient comme au printemps, à une faible immersion (vers 10-15 m). Le réchauffement des eaux superficielles en été est d'autant plus marqué que l'épaisseur de la couche de mélange est faible et que l'ensoleillement estival en 1983 a été très important.

Variations du phytoplancton; développement de Gyrodinium cf. aureolum

Le développement printanier du phytoplancton débute en mars soit bien avant l'établissement de la stratification (fig. 3c). Les teneurs en chlorophylle a sont assez peu élevées (chloro. $a<3.0 \mu \mathrm{g} \cdot \mathrm{I}^{-1}$ ) et le phytoplancton est réparti sur l'ensemble de la colonne d'eau. II est alors constitué exclusivement de diatomées (fig. 5) avec une prédominance du genre Chaetoceros. Gyrodinium cf. aureolum n'apparaît dans le système qu'au mois de mai (1000 cellules/litre en surface) véhiculé selon toute vraisemblance par les eaux dessalées qui pénètrent dans la baie à cette époque. Les diatomées sont largement majoritaires (environ 250000 cellules/litre) et Gyrodinium cf. aureolum ne repré- sente qu'une fraction réduite des dinophycées (approximativement 10\%). On peut également noter à cette époque la présence de Dinophysis acuminata (500 cellules/litre) dans les masses d'eau de la baie. Le maximum de chlorophylle $a$ atteint $5 \mu \mathrm{g} \cdot \mathrm{I}^{-1}$ (fig. 3c) et est situé au voisinage de la pycnocline. En juin, les diatomées sont largement majoritaires mais les dinophycées (100000 cellules/litre) sont représentées quasi exclusivement par Gyrodinium cf. aureolum (170 000 cellules/litre). Cette espèce prolifère au mois de juillet pour atteindre le stade d'eaux colorées (densité des cellules $>2000000$ cellules/litre). Au maximum de chlorophylle a $\left(13 \mu \mathrm{g} \cdot \mathrm{I}^{-1}\right)$, le phytoplancton est alors constitué à $95 \%$ par Gyrodinium cf. aureolum (fig. 5). La poussée n'est plus associée à la pycnocline; le maximum de chlorophylle $a$, au fur et à mesure de l'utilisation des nitrates, "plonge" vers le fond et nos résultats (fig. $3 a$ et 3c) montrent que les hautes densités en Gyrodinium cf. aureolum sont étroitement liées à la nitracline.

\section{Éléments nutritifs;}

mise en évidence d'une forte régénération en azote

Le rôle exact joué par les éléments nutritifs dans l'apparition des eaux colorées dues aux dinoflagellés demeure encore mal défini. Les études ont été souvent confinées au stade ultime du phénomène où les eaux sont colorées; ceci mène habituellement à constater l'épuisement total des masses d'eau en nutriments et à ne relever aucune corrélation entre le 

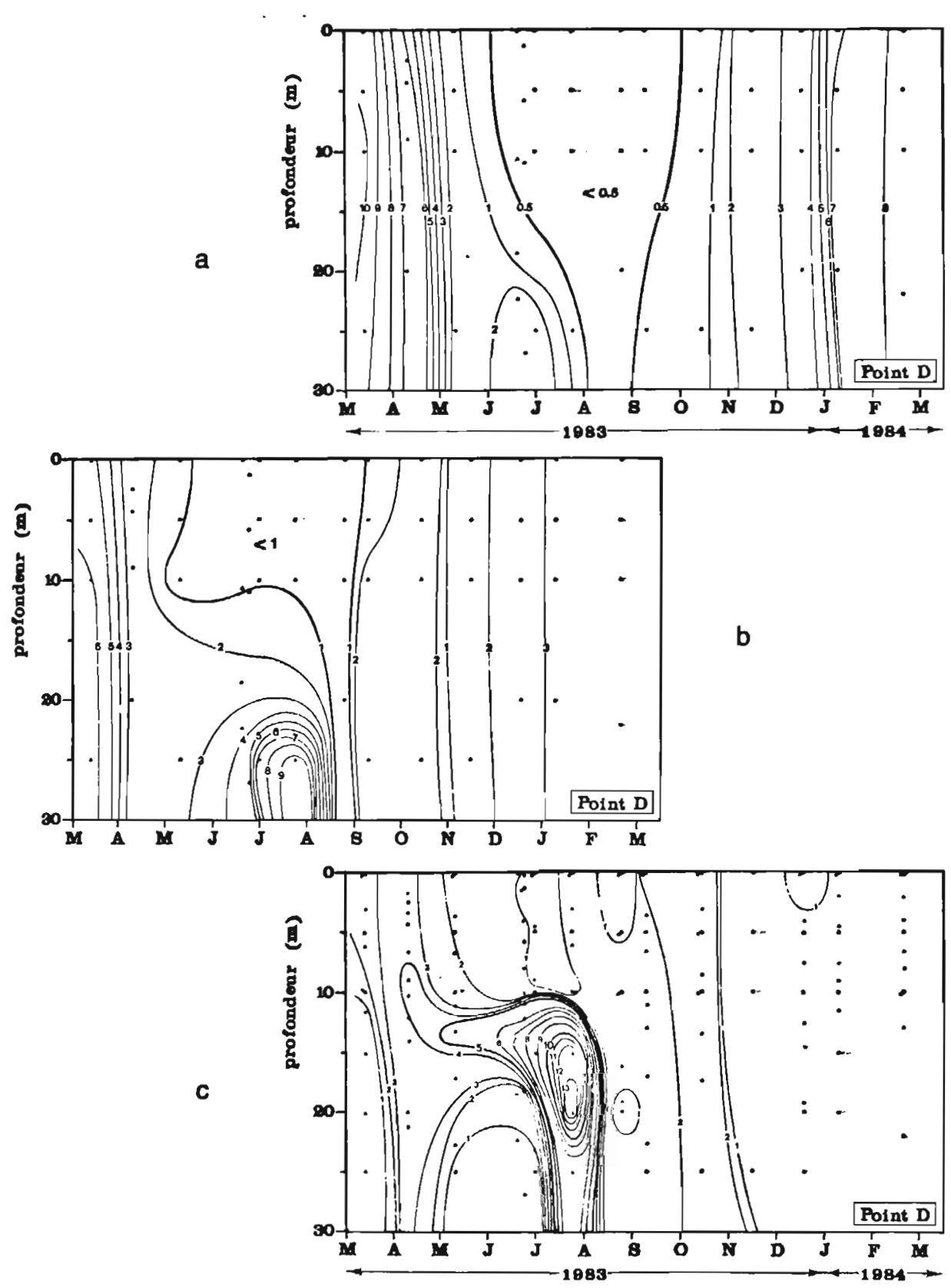

Fig. 3. - Distribution verticale en 1983 à la station D. a. nitrates (en $\mu$ atg. $\digamma^{-1}$ ). b. silicates (en $\mu \mathrm{atg} \cdot{ }^{1}{ }^{1}$ ): des fortes concentrations en silicates provenant de la régénération sont observées dans les eaux près du fond en août. c. chlorophylle a $\left(\left.\mu \mathrm{g} \cdot\right|^{-1}\right)$.

Fig. 3. - Vertical distribution in 1983 at station D. a. nitrate $\left(\mu \mathrm{g}\right.$ at $\left.\cdot \Gamma^{-1}\right)$. The whole water column is nitrate depleted in summer. b. silicate $\left(\mu \mathrm{g}\right.$ at.$\left.^{-1}\right)$. High concentrations of silicate resulting from regeneration processes are observed in bootom waters in august. c. chlorophyll a ( $\left.\mu \mathrm{g} \mathrm{I}^{1}\right)$. 

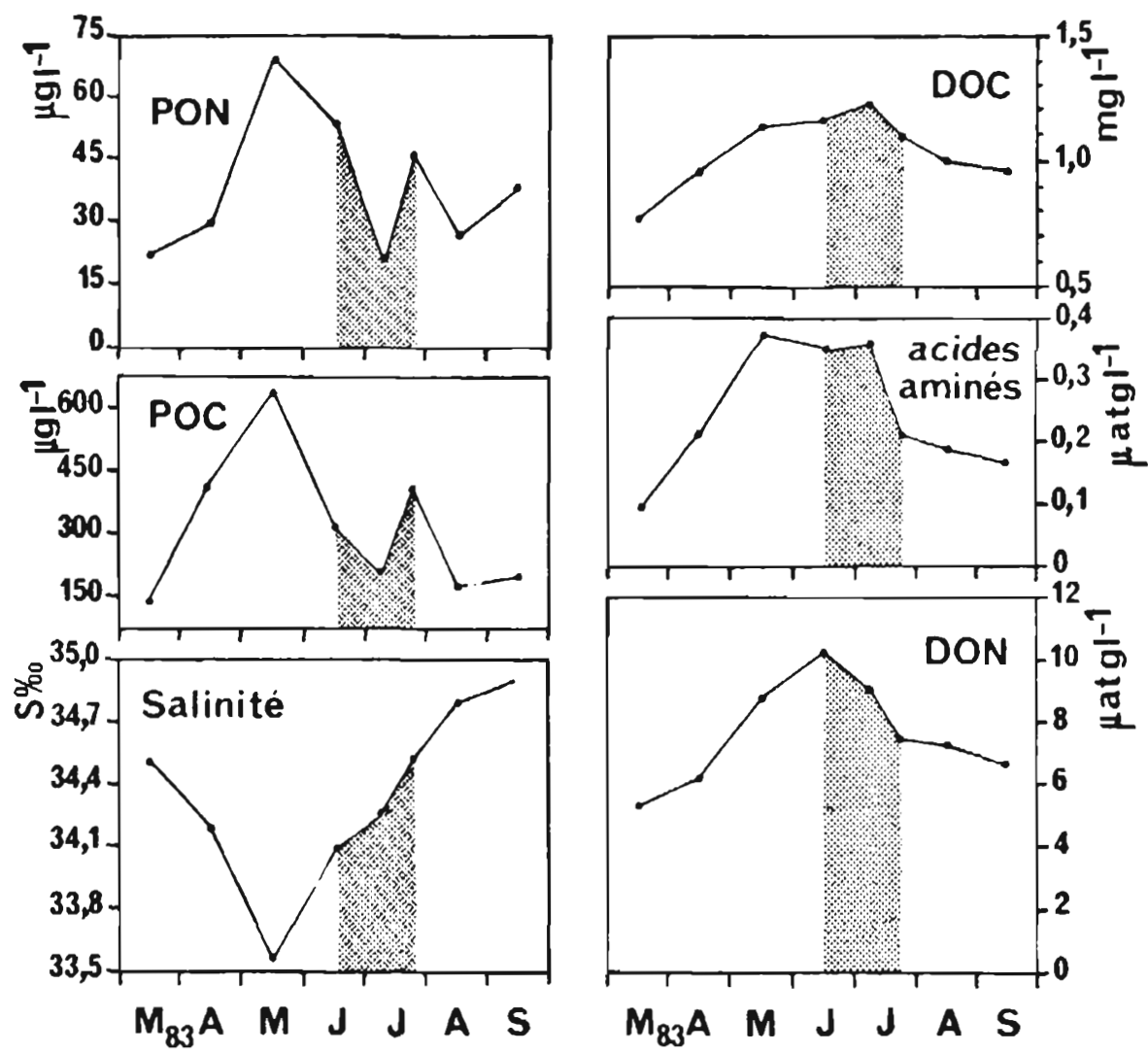

Fig. 4. - Distribution de la salinité, de la Matière Organique Particulaire et de la Matière Organique Dissoute au point D (10 premiers metres) de mars à septembre 1983.

Fig. 4. - Distribution of salinity, particulate organic matter and dissolved organic matter at station D (first ten meters) from march to september 1983.

phénomène et les teneurs en sels nutritifs. L'importance attribuée en éléments nutritifs, en fait, varie nettement selon les auteurs; les uns leur reconnaissent un rôle important (pour une revue du sujet, consulter Le Fèvre, 1986) et recherchent l'origine de la formation des eaux colorées dans un enrichissement des eaux superficielles en sels nutritifs (apports par les rivières, rejets urbains, remontées d'eaux profondes,...), d'autres leur accordent un intérêt plus limité et pensent que les processus physiques peuvent jouer un rôle important: les hautes densités en phytoplancton ne résultent pas forcément de forte production primaire et leur présence peut être liée, du moins pour partie, à la présence dans le système de méca- 


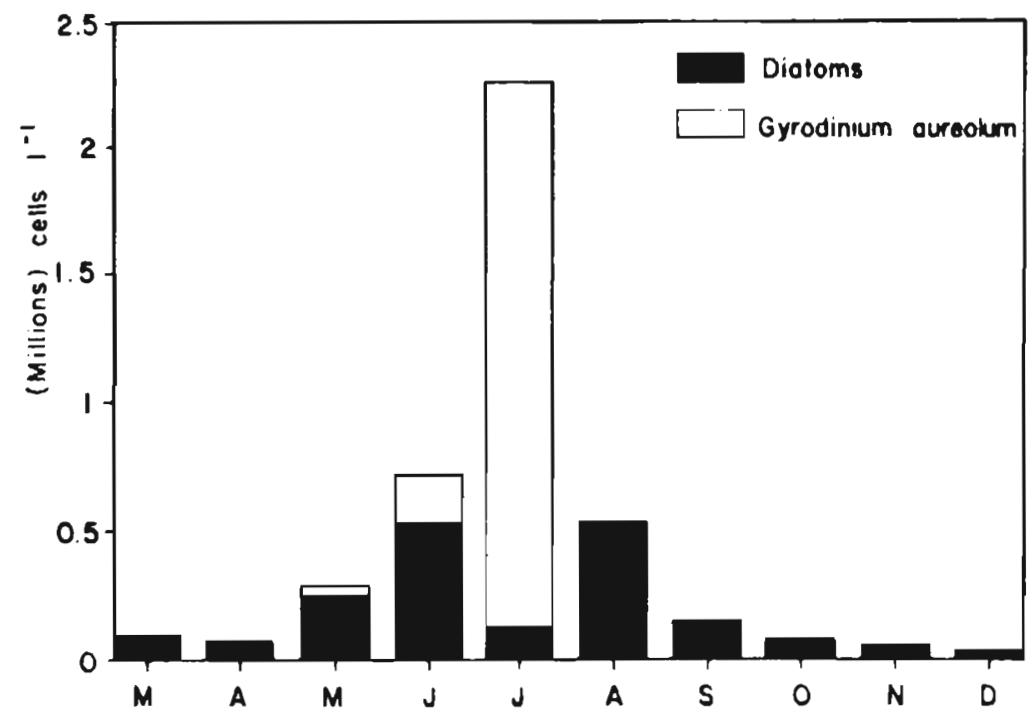

Fig. 5. - Variations saisonnières des densités des cellules de diatomées et de Gyrodinium cf. aureolum au maximum de chlorophylle $a$ à la station $D$.

Fig. 5. - Seasonal variations of the cell densities of diatoms and of Gyrodinium cf. aureolum at the chlorophyll a maximum at station $D$.

nismes physiques capables de concentrer localement les cellules phytoplanctoniques (Ryther, 1955; Rounsefell et Nelson, 1966) ou encore à la capacité des dinoflagellés de migrer verticalement dans la colonne d'eau (Eppley et al., 1968, Kamykowski, 1981). Ces migrations peuvent conduire à un regroupement des cellules dans un volume déterminé de la colonne d'eau; elles peuvent aussi permettre l'utilisation d'un stock de sels nutritifs élargi. Ce type de modèle de formation des eaux colorées où interviennent des processus de concentration mécanique des cellules nécessite comparativement moins de sels nutritifs que le modèle typique- ment "agricole" où le phytoplancton se développe sur place in situ. Nos résultats (fig. $3 a$ et $3 b$ ) apportent des précisions sur le comportement des éléments nutritifs lors des poussées de Gyrodinium cf. aureolum.

II est d'abord à remarquer que le stock hivernal de sels nutritifs est relativement modeste. Les concentrations en nitrates, silicates et phosphates sont respectivement inférieures à $10.0 \mu \mathrm{atg} \cdot \mathrm{I}^{-1}, 6.0 \mu \mathrm{atg} \cdot \mathrm{l}^{-1}$ et $0.5 \mu \mathrm{atg} \cdot \mathrm{l}^{-1}$. Les nitrites et l'ammonium présentent des teneurs réduites $\left(\mathrm{NO}_{2}^{-}<0.3 \mu \mathrm{atg} \cdot \mathrm{I}^{-1}\right.$ et $\mathrm{NH}_{4}^{\prime}<1.8 \mu$ atg..$\left.^{-1}\right)$ et le système peut être considéré, du moins en ce qui concerne les élé- 
ments nutritifs, comme exempt d'enrichissement important. L'assimilation débute au mois de mars et intéresse à cette époque de l'année l'ensemble de la colonne d'eau. Elle se poursuit en avril et dès le mois de mai, il ne subsiste que de faibles teneurs en sels nutritifs dans la colonne d'eau $\left(\mathrm{NO}_{3}^{-}<1.5 \mu\right.$ atg. $\mathrm{r}^{-1}$ et $\mathrm{Si}(\mathrm{OH})_{4}$ $<3.0 \mu$ atg $^{-1}{ }^{-1}$ ). Les eaux dessalées qui apparaissent dans le système à cette époque n'apportent que peu de sels nutritifs; il n'y a aucune perturbation apparente sur la distribution des nitrates (fig. 3a) et des silicates (fig. 3b). Ces masses d'eau appauvries en éléments nutritifs présentent par contre de fortes teneurs en matière particulaire (azote particulaire voisin de $70 \mu \mathrm{g} \cdot \mathrm{l}^{-1}$, fig. 4) qui résultent très vraisemblablement d'un développement intensif du phytoplancton alors que ces eaux se trouvaient à l'extérieur de la baie sur le plateau continental. Elles fertilisent le système en azote; la fertilisation se présente sous la forme inhabituelle d'azote organique. Le comportement des éléments nutritifs est tout à fait remarquable en été : il existe à cette époque une forte accumulation de silicates dans la couche de fond $\left(\mathrm{Si}(\mathrm{OH})_{4}>9.0 \mu \mathrm{atg} \cdot \mathrm{I}^{-}\right.$ $\left.{ }^{1}\right)$. A cet enrichissement en silicates est associée une sous-saturation marquée en oxygène (saturation en $\mathrm{O}_{2}<70 \%$ ) qui se présente comme le témoin d'une oxydation active du matériel organique et d'un recyclage abondant en sels nutritifs. On notera, et c'est un point clef des résultats, l'absence dans la "poche" de régénération de fortes teneurs en nitrates
$\left(\mathrm{NO}_{3}^{-}<2.0 \mu \mathrm{atg} \cdot{ }^{-1}\right)$. Lorsqu'on prend en compte les rapports d'oxydation de Redfield (Redfield et al., 1963), on doit s'attendre au vu du déficit en oxygène $\left(\mathrm{O}_{2}>2 \mathrm{ml} \cdot \mathrm{I}^{-1}\right)$ à des teneurs élevées en nitrates ou en ammonium dans la couche de fond ( $\mathrm{N}$ minéral $\left.>10.0 \mu \mathrm{atg} \cdot \mathrm{I}^{-1}\right)$. Ce n'est pas le cas et il est tout à fait vraisemblable que l'azote régénéré a été utilisé lors de la poussée de Gyrodinium cf. aureolum. Le recyclage apparaît dans la Baie de Douarnenez comme une source "discrète" mais très efficace d'azote minéral. II est probable que cette régénération est entretenue, du moins pour partie, par les matières particulaires importées par le système au printemps. Ces résultats nous ont convaincu de la nécessité de mesurer de manière directe par la technique ${ }^{15} \mathrm{~N}$ les flux d'azote associés au développement de Gyrodinium cf. aureolum. Les premières mesures ont été effectuées en 1986 sur le front d'Ouessant (fig. 1). On trouvera ciaprès une présentation préliminaire de ces résultats.

\section{Conditions de développement de} Gyrodinium cf. aureolum au voisinage du front thermique d'Ouessant (1986); Mesures des flux d'azote

\section{Description du phénomène}

\section{Structure thermique}

Le front thermique d'Ouessant est établi de manière stable en été à l'en- 

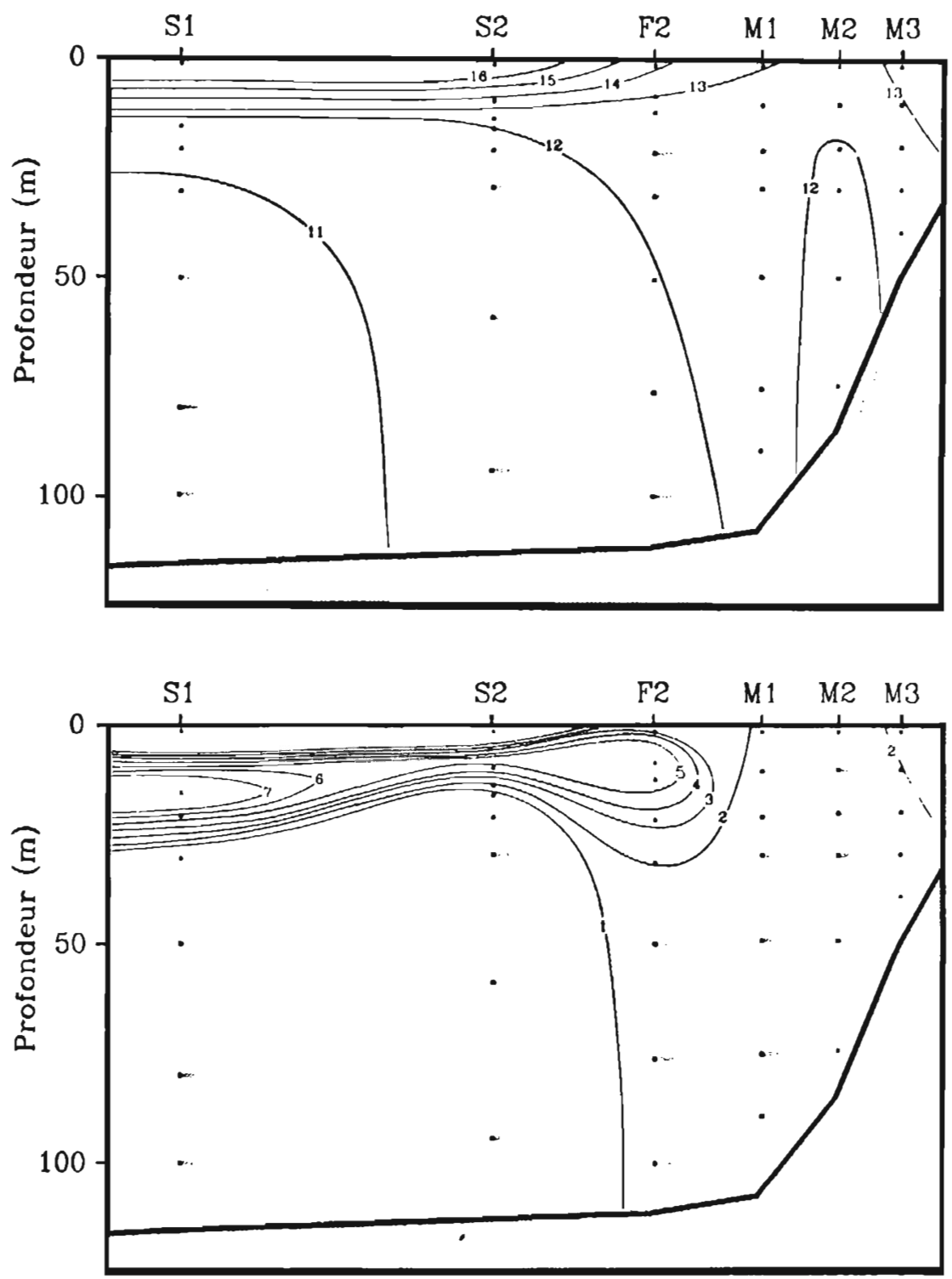

Fig. 6. - Distribution verticale dans la zone du Front d'Ouessant en juillet 1986: a. température $\left({ }^{\circ} \mathrm{C}\right)$. b. chlorophylle a $\left(\mu \mathrm{g} \cdot{ }^{-1}\right)$.

Fig. 6. - Vertical distribution in the Ushant frontal zone in July 1986 : a. temperature $\left({ }^{\circ} \mathrm{C}\right)$. b. chlorophyll a $\left(\mu \mathrm{g} \cdot \mathrm{l}^{-1}\right)$. 
trée de la Manche (fig. 1). La radiale étudiée (fig. 6) coupe le front au Nord d'Ouessant; la structure thermique est tout à fait classique et on peut distinguer (fig. 6a):

- un secteur côtier avec des eaux relativement froides $\left(12\right.$ à $\left.13^{\circ} \mathrm{C}\right)$; la colonne d'eau est thermiquement homogène en raison d'un fort brassage lié à l'existence de courants de marée très intenses;

- au large (stations $S_{1}$ et $S_{2}$ ) un domaine stratifié; une thermocline bien marquée sépare une couche de surface où la température est relativement élevée $\left(16\right.$ à $\left.17^{\circ} \mathrm{C}\right)$ d'une couche profonde plus froide $\left(11^{\circ} \mathrm{C}\right.$ environ) ;

- entre ces deux secteurs, dans la zone où la thermocline remonte vers la surface, le front thermique à proprement parler.

\section{Distribution verticale \\ de la chlorophylle}

Le domaine homogène est caractérisé par une distribution relativement uniforme de la chlorophylle a de la surface au fond (fig. 6b); les concentrations sont peu élevées (chloro. $a<2.0 \mu \mathrm{g} \cdot \mathrm{l}^{-1}$ ). Intégrées sur la colonne d'eau, elles représentent cependant une biomasse phytoplanctonique importante. Dans ce domaine, le phytoplancton est composé essentiellement de diatomées, les genres Leptocylindrus et Nitzchia étant les plus abondants. Dans le secteur le plus au large (station $S_{1}$ ), des teneurs élevées en chlorophylle a $\left.\left(>7.0 \mu \mathrm{g}^{-1}\right)^{-1}\right)$ sont mesurées à la profondeur de la thermocline, juste au-dessus de la nitracline. Ces fortes concentrations correspondent au développement de l'espèce Gyrodinium cf. aureolum qui atteint une densité supérieure à deux millions de cellules par litre au maximum de chlorophylle a. Elles sont associées à des sursaturations en oxygène dissous supérieures à $125 \%$ (L'Helguen, 1991) qui traduisent une intense activité photosynthétique; ceci semble indiquer que le développement important de Gyrodinium cf. aureolum s'est effectué sur place.

\section{Sources d'azote pour le développement de Gyrodinium cf. aureolum; mesures au ${ }^{15} \mathrm{~N}$}

\section{Assimilation des nitrates et de l'ammonium (Station $S_{1}$ )}

Les variations verticales des taux d'absorption suivent celles des teneurs en chlorophylle a (fig. 7). Les taux d'absorption maxima sont mesurés en subsurface à la profondeur de la thermocline; ils sont associés au développement intense de Gyrodinium cf. aureolum. La majeure partie de l'azote utilisé à ce niveau est sous la forme d'ammonium; aux plus fortes densités de Gyrodinium cf. aureolum, l'absorption de l'ammonium représente plus de $90 \%$ de l'absorption azotée totale.

\section{Régénération de l'ammonium (Station $S_{1}$ )}

La régénération de l'ammonium est particulièrement intense à la station $S_{1}$ (fig. 8); les taux de régénération 


\section{Station S1}
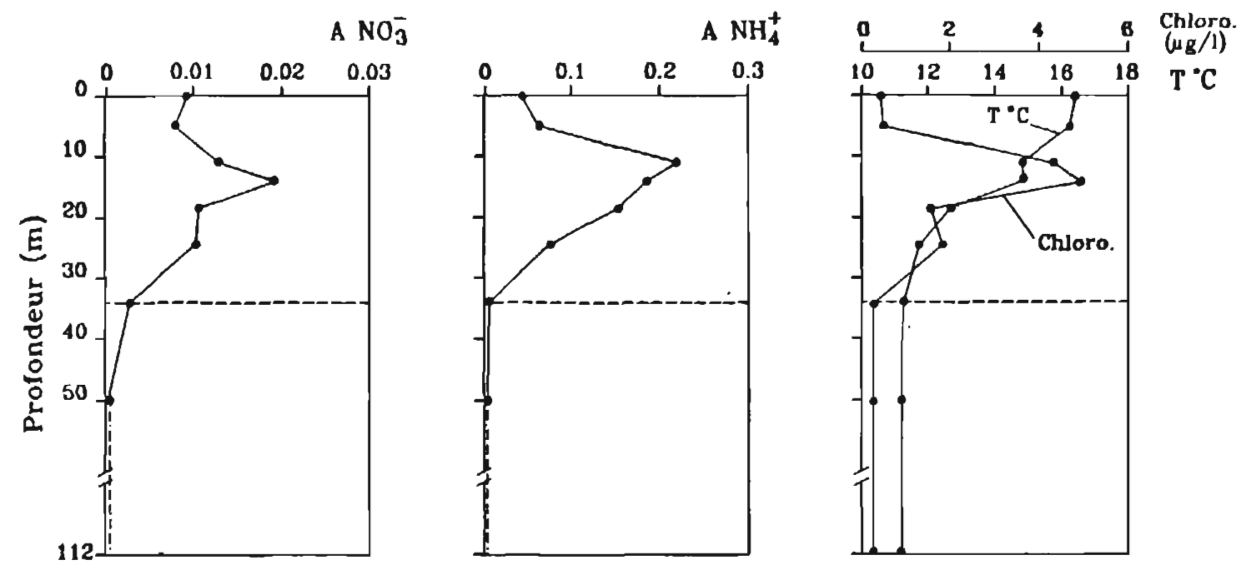

Fig. 7. - Variations verticales des taux d'absorption des nitrates et de l'ammonium (exprimés en $\mu$ moles $\mathrm{N} \cdot \mathrm{I}^{-1} \cdot \mathrm{h}^{-1}$ ), de la temperature et de la chlorophylle a à la station $S_{1}$ en juillet 1986 (en pointillé, la profondeur de la couche euphotique).

Fig. 7. - Vertical variations of nitrate and ammonium absorption rates (expressed as $\mu$ moles $\mathrm{N} \cdot \mathrm{I}^{-1} \cdot \mathrm{h}^{-1}$ ), of temperature and chlorophyll $a$ at station $\mathrm{S}_{1}$ in july 1986 (in dotted line, the depth of the euphotic zone).

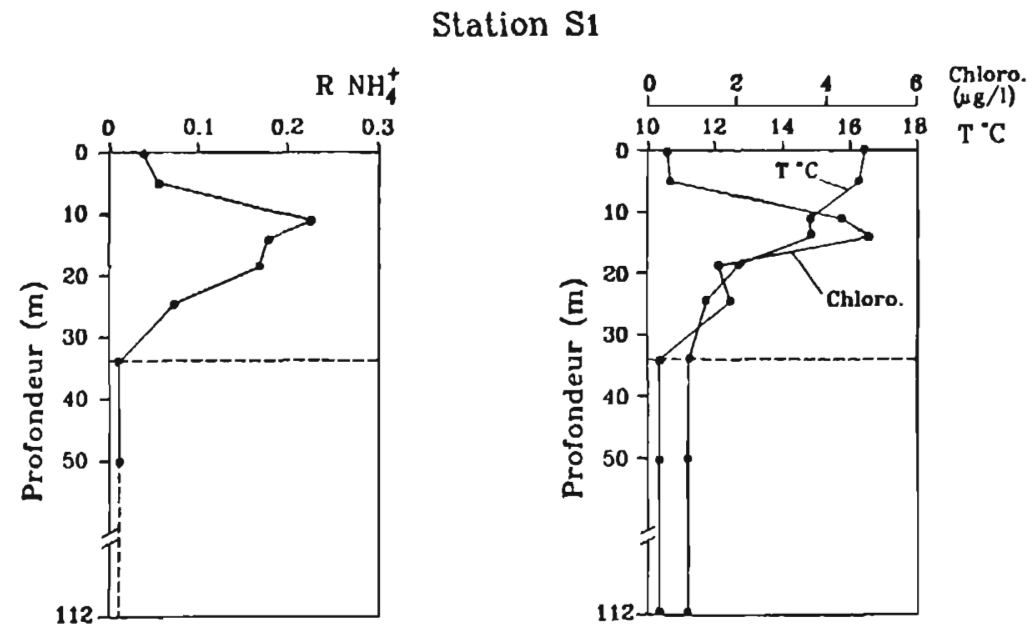

Fig. 8. - Variations verticales des taux de régénération de l'ammonium (en $\mu$ moles $\mathrm{N}-\mathrm{NH}_{4}^{+} \cdot \mathrm{I}^{-1} \cdot \mathrm{h}^{-1}$ ), de la température et de la chlorophylle a à la station $S_{1}$ en juillet 1986 (en pointillé, la profondeur de la couche euphotique).

Fig. 8. - Vertical variations of ammonium regeneration rates (expressed as $\mu$ moles $\mathrm{N}-\mathrm{NH}_{4}^{+} \cdot \Gamma^{-1} \cdot \mathrm{h}^{-1}$ ), of temperature and chlorophyll $a$ at station $S_{1}$ in july 1986 (in dotted line, the depth of the euphotic zone). 
atteignent $0.25 \mu$ moles $\mathrm{N}^{-N_{4}^{+}}$à la profondeur de la thermocline et sont suffisants pour assurer les besoins en ammonium de Gyrodinium cf. aureolum. La régénération intense de l'ammonium est associée à un stock important de matériel détritique concentré à la profondeur de la thermocline. L'origine de cette matière organique n'est pas connue de manière précise: elle a été attribuée à une sédimentation des diatomées qui se développent en abondance sous certaines conditions dans la zone de balancement du front (L'Helguen, 1986) et qui sont, de manière générale, peu exploitées par le zooplancton (Le Fèvre, 1986).

\section{CONCLUSION}

Nos résultats sur le front d'Ouessant montrent clairement que l'azote minéral nécessaire au développement de Gyrodinium cf. aureolum résulte de phénomènes intenses de régénération qui se développent sur place. Ils sont en accord avec les résultats antérieurs obtenus sur le front interne de l'Iroise (Baie de Douarnenez). Dans le premier cas, la régénération est induite par la présence, en abondance, de matière organique particulaire fournie sur place; la prolifération de Gyrodinium cf. aureolum correspond à un phénomène naturel. Dans le second cas, le matériel détritique est introduit dans le système qui est alors perturbé dans son fonctionnement. Contrairement aux hypothèses généralement proposées, le mécanisme mis en avant n'implique pas le trans- fert vertical marqué de nitrates à partir de la couche de fond; il ne nécessite pas non plus une migration verticale des cellules.

\section{BIBLIOGRAPHIE}

Ayres P.A., Seaton D.D. \& Tett P.B., 1982. Plankton blooms of economic importance to fisheries in UK waters 19681982. ICES CM 1982/L: 38 (mimeo).

Ballantine D. \& Smith E.M., 1973. Observations on blooms of the dinoflagellate Gyrodinium aureolum Hulburt in the River Conway and its occurence along the North Wales coast. Br. Phycol. J., 8: 233-238.

Birrien J.L., 1987. Cycles des variations des éléments nutritifs et du phytoplancton en Baie de Douarnenez et dans les secteurs adjacents: importance du front côtier de l'Iroise. These de Doctorat de I'Université de Bretagne Occidentale, Brest.

Birrien J.L., Wafar M.V.M., Le Corre P. \& Riso R., 1991. Nutrients and primary production in a shallow stratified ecosystem in the Iroise Sea. J. Plankt. Res., 13: 721-742.

Boalch G.T., 1979. The dinoflagellate bloom on the coast of south west England, August-September 1978. J. mar. biol. Ass. U.K., 59: 515-517.

Braarud T. \& Heimdal B.R., 1970. Brown water on the Norwegian coast in autumn 1966. Nytt Mag. Bot., 17: 91-97.

Dahl E., Danielssen D.S. \& Bohle B., 1982. Mass occurence of Gyrodinium aureolum Hulburt and fish mortality along the southern coast of Norway in September-October 1981. ICES CM 1982/L: 56 (mimeo).

Eppley R.W., Holm Hansen O. \& Strickland J.D.H., 1968. Some observations on the vertical migration of dinoflagellates. J. Phycol., 4: 57-64. 
Foster G.R., 1979. Mortality of the bottom fauna and fish in St Austell Bay and neighbouring areas. J. mar. biol. Ass. U.K., 59: 517-520.

Grall J.R., 1977. Sur une "eau colorée" à Gyrodinium aureolum Hulburt observée en Manche. Trav. Stn biol. Roscoff, 23: 19-22.

Griffiths A.B., Dennis P. \& Potts G.W., 1979. Mortality associated with a phytoplankton bloom off Penzance in Mounts Bay. J. mar. biol. Ass. U.K., 59: $520-521$.

Helm M.M., Hepper B.T., Spencer B.E. \& Walne P.R., 1974. Lugworm mortalities and a bloom of Gyrodinium aureolum Hulburt in the eastern Irish Sea, autumn 1971. J. mar. biol. Ass. U.K., 54: 857-869.

Holligan P.M., 1979. Dinoflagellate blooms associated with tidal fronts around the British Isles. Toxic Dinoflagellate Blooms. D.C. Taylor and H.H. Seliger Ed.: 249-256. Elsevier. New York.

Hulburt E.M., 1957. The taxonomy of unarmored Dinophyceae of shallow embayments on Cape Cod, Massa- ssuchetts. Biol. Bull. mar. biol. Lab., Woods Hole 112: 196-219.

Kamykowski D., 1981. Laboratory experiments on the diurnal vertical migration of marine dinoflagellates through temperature gradients. Mar. Biol., 62: 5764.

Lassus P., 1984. Bilan des phénomènes d'eaux colorées et des perturbations observées dans le phytoplancton côtier pour l'année 1983. Rapport scientifique ISTPM, Nantes, $27 \mathrm{p}$.

Le Fèvre J., 1986. Aspects of the biology of frontal systems. Adv. mar. Biol., 23: 163-299.

Le Fevre J. \& Grall J.R., 1970. On the relationship of Noctiluca swarming off the western coast of Brittany with hydrological features and plankton characteristics of the environment. J. exp. mar. Biol. Ecol., 4: 287-306.

L'Helguen S., 1991. Absorption et régénération de l'azote dans les écosystèmes pélagiques du plateau continental de la Manche Occidentale. Relation avec le régime de mélange vertical des masses d'eaux; Cas du front thermique d'Ouessant. Thèse de Doctorat d'Université, Université de Bretagne Occidentale, Brest, $212 \mathrm{p}$.

Lindahl O., 1983. On the develompment of a Gyrodinium aureolum occurence on the Swedish coast in 1982. Mar. Biol., 77: 143-150.

Lorenzen C.J., 1966. A method for the continuous measurements of in vivo chlorophyll concentration. Deep-Sea Res., 13: 223-227.

Margalef R., Estrada M. \& Blasco D., 1979. Functional morphology of organisms involved in red tides, as adapted to decaying turbulence. Toxic Dinoflagellate Blooms. D.L. Taylor and H.H. Seliger ed.: 84-93, Elsevier, New York.

Morin P., Birrien J.L., Le Corre P., 1989. The frontal systems in the Iroise Sea: development of Gyrodinium aureolum Hulburt in the inner front. Scient. Mar., 53: 103-111.

Ottway B., Parker M., Mac Grath D. \& Crowley M., 1979. Observations on a bloom of Gyrodinium aureolum Hulburt on the south coast of Ireland, summer 1976, associated with mortalities of littoral and sublittoral organisms. Ir. Fish. Inv. (B), 18: 9 p.

Parker M., Dunne T. \& Mc Ardle J., 1982. Exceptional marine blooms in Irish coastal waters. ICES CM 1982/L: 44 (mimeo).

Partensky F. \& Sournia A., 1986. Le dinoflagellé Gyrodinium aureolum dans le plancton de l'Atlantique Nord: identification, écologie, toxicité. Cryptogamie, Algologie, 7: 251-275.

Pingree R.D., Holligan P.M. \& Head R.N., 1977. Survival of dinoflagellate blooms in the western English Channe. Nature, London, 258: 266-269.

Pingree R.D., Pugh P.R., Holligan P.M. \& Forster G.R., 1975. Summer phytoplankton blooms and red tides along tidal fronts in the approaches to the 
English Channel. Nature, London, 258: 672-677.

Pybus C., 1980. Observations on a Gyrodinium aureolum (dinophyta) bloom off the south coast of Ireland. J. mar. biol. Ass. U.K., 60: 661-674.

Redfield A.C., Ketchum B.H. \& Richards F.A., 1963. The influence of organisms on the composition of sea-water. In: The Sea, Vol. 2: 26-27, (M.N. Hill, Ed.), New-York, London.

Ryther J.H., 1955. Ecology of autotrophic dinoflagellates with reference to red water conditions. In: The luminescence of biological systems, 41: 387414, (F.H. Johnson, Ed.), Washington.

Rounsefell G.A. \& Nelson W.R., 1966. Red tide research summarized to 1964 including an annotated bibliography. Spec. Sci. Rep. U.S. Fish. wild. Serv. Fish, 535: $85 \mathrm{p}$.

Ryther J.H., 1955. Marine dinoflagellate with reference to red water conditions in the luminescence of biological systems. The luminescence of biological systems. F.H. Johnson Ed.: 387-414.

Tangen K., 1977. Blooms of Gyrodinium aureolum (Dinophyceae) in north European waters, accompanied by mortality in marine organisms. Sarsia, 63: 123-133.

Tréguer P. \& Le Corre P., 1975. Manuel d'analyse des sels nutritifs dans l'eau de mer (utilisation de l'autoanalyser II Technicon), $2^{ø}$ édition, Université de Bretagne Occidentale, Brest, $110 \mathrm{p}$.

Widdows J., Moore M.MN, Lowe D.M. \& Salheld P.N., 1979. Some effects of a dinoflagellate bloom (Gyrodinium aureolum) on the mussel, Mytilus edulis. J. mar. biol. Ass. U.K., 59: 522-524.

Yentsch C.S. \& Menzel D.W., 1963. A method for the determination of phytoplankton chlorophyll and phaeophytin by fluorescence. Deep-Sea Res., 10: 221-231. 